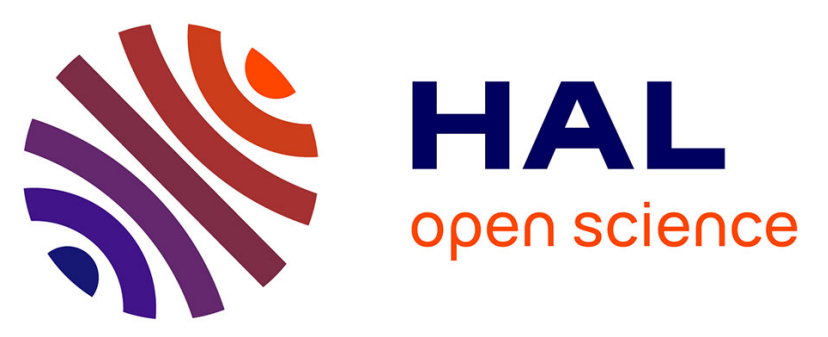

\title{
On the Extension of the Product Model in Polsar Processing for Unsupervised Classification Using Information Geometry of Covariance Matrices
}

Pierre Formont, Jean-Philippe Ovarlez, Frédéric Pascal, Gabriel Vasile, Laurent Ferro-Famil

\section{To cite this version:}

Pierre Formont, Jean-Philippe Ovarlez, Frédéric Pascal, Gabriel Vasile, Laurent Ferro-Famil. On the Extension of the Product Model in Polsar Processing for Unsupervised Classification Using Information Geometry of Covariance Matrices. IGARSS 2011 - IEEE International Geoscience and Remote Sensing Symposium, Jul 2011, Vancouver, Canada. pp.1361-1364, 10.1109/IGARSS.2011.6049318 . hal00640872

\section{HAL Id: hal-00640872 https://hal.science/hal-00640872}

Submitted on 14 Nov 2011

HAL is a multi-disciplinary open access archive for the deposit and dissemination of scientific research documents, whether they are published or not. The documents may come from teaching and research institutions in France or abroad, or from public or private research centers.
L'archive ouverte pluridisciplinaire HAL, est destinée au dépôt et à la diffusion de documents scientifiques de niveau recherche, publiés ou non, émanant des établissements d'enseignement et de recherche français ou étrangers, des laboratoires publics ou privés. 


\title{
ON THE EXTENSION OF THE PRODUCT MODEL IN POLSAR PROCESSING FOR UNSUPERVISED CLASSIFICATION USING INFORMATION GEOMETRY OF COVARIANCE MATRICES
}

\author{
P. Formont ${ }^{1,2}$, J.P. Ovarlez ${ }^{1,2}$, F. Pascal ${ }^{2}$, G. Vasile $^{3}$ and L. Ferro-Famil ${ }^{4}$ \\ ${ }^{1}$ : French Aerospace Lab, ONERA, France, jean-philippe.ovarlez@onera.fr \\ ${ }^{2}$ : SONDRA, Supélec, France, pierre.formont@supelec.fr,frederic.pascal@supelec.fr \\ ${ }^{3}$ : GIPSA Lab, CNRS, France, gabriel.vasile@gipsa-lab.grenoble-inp.fr \\ ${ }^{4}$ : IETR, SAPHIR, France, laurent.ferro-famil@univ-rennes1.fr
}

\begin{abstract}
We discuss in the paper the use of the Riemannian mean given by the differential geometric tools. This geometric mean is used in this paper for computing the centers of class in the polarimetric $\mathrm{H} / \alpha$ unsupervised classification process. We can show that the centers of class will remain more stable during the iteration process, leading to a different interpretation of the $H / \alpha / A$ classification. This technique can be applied both on classical SCM and on Fixed Point covariance matrices. Used jointly with the Fixed Point CM estimate, this technique can give nice results when dealing with high resolution and highly textured polarimetric SAR images classification.
\end{abstract}

Index Terms - SAR, Polarimetry, Classification, Estimation, Differential Geometry.

\section{INTRODUCTION}

The recently launched POLSAR systems are now capable of producing high quality polarimetric SAR images of the Earth surface under meter resolution. The additional polarimetric information allows the discrimination of different scattering mechanisms. In [1] was introduced the entropy-alphaanisotropy $(\mathrm{H} / \alpha / \mathrm{A})$ classification based on the eigenvalues of the polarimetric (or coherency) Covariance Matrix (CM). This CM is usually estimated, under homogeneous and Gaussian assumptions, with the well known Sample Covariance Matrix (SCM) which is Wishart distributed. Based on this decomposition, unsupervised classification of SAR images can be performed by an iterative algorithm based on a complex Wishart density function. It uses the $\mathrm{H} / \alpha$ decomposition results to get an initial segmentation into clusters, then the Kmeans clustering is implemented by considering the polarimetric $\mathrm{CM}$ as the feature vectors. This technique needs however to derive by a Euclidian mean operation the averaged CM of each class and to compute by Wishart distance the mininal distance between each pixel CM and with all the class centers.

The decrease of the resolution cell leads however to more complicated effects like spatial heterogeneity or non Gaus- sianity. Hence, some areas (grass, trees, ...) usually considered as random backscattering mechanisms can become punctual deterministic backscattering mechanisms. The usual techniques of classification, detection, speckle filtering, used for decametric resolution have to be adapted to these new challenging problems. Recent studies have shown that the spatial heterogeneity of the observed scene allows for nonGaussian clutter modelling. One commonly used fully polarimetric non-Gaussian clutter model is the compound Gaussian model: the polarimetric clutter information $m$-vector $\mathbf{k}$ is modeled as a SIRV (Spherically Invariant Random Vector), i.e. the product between the square root of a scalar random variable $\tau$ (called the texture) and an independent, zero mean, complex circular Gaussian random vector $\mathbf{z}$ (called the polarimetric speckle) and characterized by an unknown zero-mean and circular CM M:

$$
\mathbf{k}=\sqrt{\tau} \mathbf{z}
$$

In this model, $\tau$ can represent the spatial variation of the intensity of the wave vector $\mathbf{k}$ from pixel to pixel. All the polarimetric information (phase relationships within the wave vector) is then contained only in the normalized CM M $(\operatorname{Tr}(\mathbf{M})=m$ where $\operatorname{Tr}($.$) is the trace operator). Condition-$ ally to a given pixel (equivalently to a given $\tau$ ), the wave vector is then fully Gaussian distributed.

The aim of this proposed paper is twofold. Firstly, we propose in this paper to briefly recall original results obtained recently in [2] for the joint Maximum Likelihood estimation of the texture and the polarimetric CM. These results, based on the above Fixed Point CM estimate, allowed to derive a new distance for SIRV CM and to propose a new technique of speckle filtering in heterogeneous environment. Secondly, we introduce a metric-based mean for the space of positivedefinite Hermitian CM. An emerging theory $[3,4,5]$ allows to take into account the fact that Euclidian representation cannot describe the space of positive-definite Hermitian CM. 


\section{ESTIMATION OF THE COHERENCY COVARIANCE MATRIX}

\subsection{Gaussian homogeneous case}

In homogeneous and Gaussian clutter assumption, the texture $\tau$ is assumed to be constant and the same for all pixels. In that case, the statistic of the secondary data is Gaussian and the $\mathrm{CM}$ can be estimated by Maximum Likelihood (ML) theory with a set of $N$ secondary data $\mathbf{k}_{n}, n \in[1, N]$ as:

$$
\widehat{\mathbf{M}}_{S C M}=\frac{1}{N} \sum_{n=1}^{N} \mathbf{k}_{n} \mathbf{k}_{n}^{H} .
$$

This unbiased CM estimate is Hermitian, Wishart-distributed with $N$ degrees of freedom. Another well known POLSAR parameter maximizing the mean-to-variance ratio is the Polarimetric Whitening Filter (PWF) as $\mathrm{PWF}_{\mathrm{SCM}}=$ $\mathbf{k}_{n}^{H} \widehat{\mathbf{M}}_{S C M}^{-1} \mathbf{k}_{n}$. For supervised and unsupervised POLSAR data clustering in the Gaussian case, a LR distance $D_{W}$ between a given pixel $i$ characterized by its SCM $\widehat{M}^{i}$ and a class center characterized by the SCM $\widehat{M}_{\omega}$ has been derived [6], [7] :

$$
D_{W}\left(\widehat{\mathbf{M}}_{i}, \widehat{\mathbf{M}}_{\omega}\right)=\ln \frac{\left|\widehat{\mathbf{M}}_{\omega}\right|}{\left|\widehat{\mathbf{M}}_{i}\right|}+\operatorname{Tr}\left(\widehat{\mathbf{M}}_{\omega}^{-1} \widehat{\mathbf{M}}_{i}\right) .
$$

When dealing with heterogeneous and/or non-Gaussian clutter, the SCM is no longer robust. Under SIRV assumption, the SCM takes the form:

$$
\widehat{\mathbf{M}}_{S C M}=\frac{1}{N} \sum_{n=1}^{N} \tau_{n} \mathbf{z}_{n} \mathbf{z}_{n}^{H} .
$$

The presence of all textures $\tau_{n}$ of the $N$ secondary wave vectors $k_{n}$ makes this estimate strongly polluted.

Figure 1 presents the dataset used for the discussion (polarimetric SAR images of ONERA SETHI system in Bretigny in France) and the conventional $\mathrm{H} / \alpha$ classification using SCM. We can see clearly that the conventional classification is strongly connected to the intensity variation of the RGB Pauli representation. It can be explained by the fact that the SCM (and so, the Wishart distance, the centers of class) is dependent on the intensity (or texture) present in the reference cells. Figure 2 shows, after 10 iterations, the locations of all the pixels in the $\mathrm{H}-\alpha$ plan (for height classes). The distribution of pixels belonging to a given class seems very unordered.

\subsection{Non-Gaussian and Heterogeneous Case}

In the SIRV model, the CM is generally an unknown parameter which can be estimated from Maximum Likelihood (ML) Theory. In [8], Gini et al. derived the ML estimate $\widehat{\mathbf{M}}_{F P}$ of the covariance matrix $\mathbf{M}$ for deterministic texture, which is

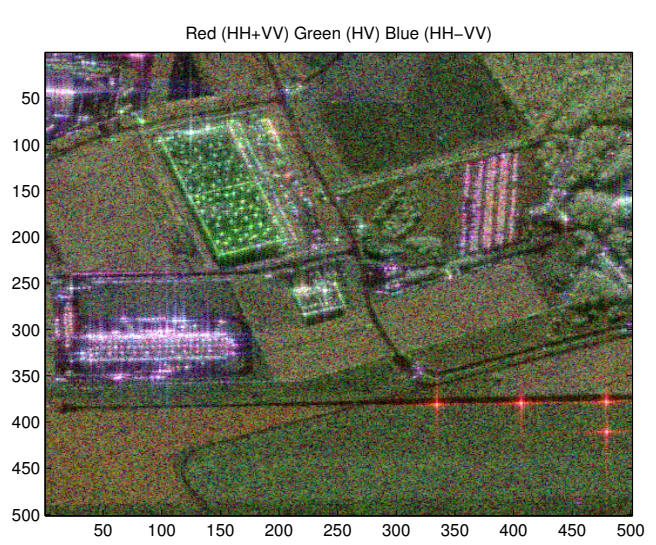

(a) ONERA SETHI polarimetric SAR Image in RGB Pauli basis

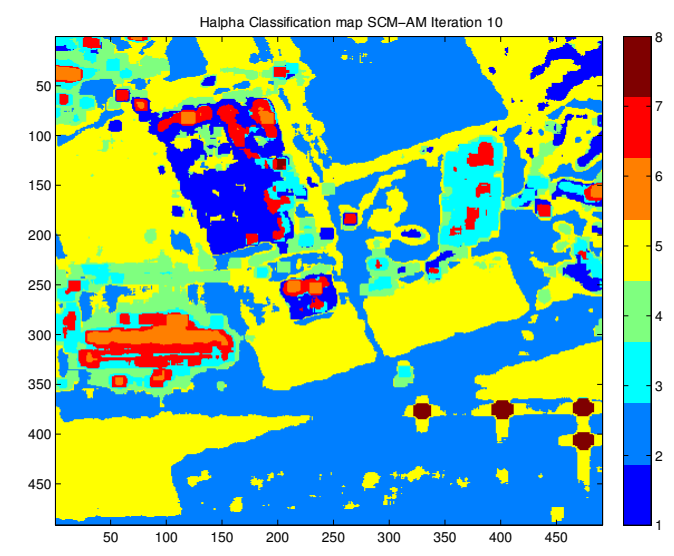

(b) Conventional $\mathrm{H} / \alpha$ classification after 10 iterations

Fig. 1. Comparison between Pauli representation and conventional unsupervised $\mathrm{H} / \alpha$ classification after 10 iterations.

the solution of the following equation:

$$
\widehat{\mathbf{M}}_{F P}=f(\mathbf{M})=\frac{m}{N} \sum_{i=1}^{N} \frac{\mathbf{k}_{i} \mathbf{k}_{i}^{H}}{\mathbf{k}_{i}^{H} \widehat{\mathbf{M}}_{F P}^{-1} \mathbf{k}_{i}} .
$$

This approach has been used in [9] by Conte et al. to derive a recursive algorithm for estimating the solution matrix $\mathbf{M}_{F P}$ called the Fixed Point Covariance matrix. It has been shown in [8] and [9] that the estimation scheme from (5), developed under the deterministic texture case, yields also an Approximate ML (AML) estimator under stochastic texture hypothesis. This study has been completed by the work of Pascal et al. [10], which recently established the existence and the uniqueness of the Fixed Point estimate, as well as the convergence of the recursive algorithm whatever the initialization. This matrix estimate is shown to be robust, unbiased, consistent and asymptotically distributed as Gaussian PDF [11]. One could suppose legitimately that it has the same behavior as a Wishart distributed matrix with $m N /(m+1)$ degrees of freedom. The generalized ML estimator for the $\tau_{n}$ texture for 


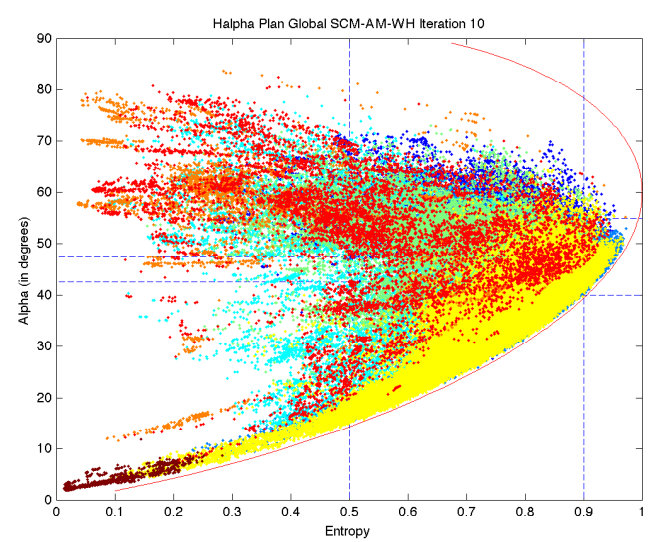

Fig. 2. Location of the pixels in the $\mathrm{H} / \alpha$ plan after 10 iterations in the Gaussian case.

the primary data $\mathbf{k}_{n}$ is given by:

$$
\widehat{\tau}_{n}=\frac{\mathbf{k}_{n}^{H} \widehat{\mathbf{M}}_{F P}^{-1} \mathbf{k}_{n}}{m},
$$

which is proportional to the SIRV Polarimetric Whitening Filter $\mathrm{PWF}_{\mathrm{SIRV}}=\mathbf{k}_{n}^{H} \widehat{\mathbf{M}}_{F P}^{-1} \mathbf{k}_{n}$. The role of the matrix $\widehat{\mathbf{M}}_{F P}$ is here to whiten the possible correlation existing within the polarimetric channels without being polluted by the power of the secondary data used to estimate this matrix. A new LR SIRV distance $D_{S}$ between two FP CM $\widehat{\mathbf{M}}_{i}$ and $\widehat{\mathbf{M}}_{\omega}$ has also been derived in [2]:

$$
D_{S}\left(\widehat{\mathbf{M}}_{i}, \widehat{\mathbf{M}}_{\omega}\right)=\ln \frac{\left|\widehat{\mathbf{M}}_{\omega}\right|}{\left|\widehat{\mathbf{M}}_{i}\right|}+\frac{m}{N} \sum_{n=1}^{N} \frac{\mathbf{k}_{n}^{H} \widehat{\mathbf{M}}_{\omega}^{-1} \mathbf{k}_{n}}{\mathbf{k}_{n}^{H} \widehat{\mathbf{M}}_{i}^{-1} \mathbf{k}_{n}} .
$$

This equation depends on the secondary data $\left(\mathbf{k}_{n}\right)_{n=1, N}$ but can nicely be simplified as:

$$
D_{S}\left(\widehat{\mathbf{M}}_{i}, \widehat{\mathbf{M}}_{\omega}\right)=D_{W}\left(\widehat{\mathbf{M}}_{i}, \widehat{\mathbf{M}}_{\omega}\right) .
$$

This result shows that, again, the FP CM could be considered as a Wishart CM. Figure 3 shows, after 10 iterations, the locations of all the pixels in the $\mathrm{H}-\alpha$ plane (for eight classes). The distribution of pixels belonging to a given class seems much better ordered.

\section{RIEMANNIAN DISTANCE}

Rigorously, the averaged $\mathrm{CM} \mathrm{M}_{\omega_{l}}$ (SCM or Fixed Point) of a $\mathrm{H} / \alpha /$ A cluster $l$ cannot be computed with the Euclidean metric, i.e. usual arithmetic mean as $\mathbf{M}_{\omega_{l}}=1 / K \sum_{k=1}^{K} \mathbf{M}_{k}^{l}$ where $\mathbf{M}_{k}^{l}, k \in[1, K]$ are the $K \mathbf{C M}$ of all pixels belonging to the class $\omega_{l}$ in the $\mathrm{H} / \alpha$ plan. It is well known that after a few iterations of the unsupervised classification, all the class centers move significantly within the $\mathrm{H} / \alpha$ plane, leading to a more difficult physical interpretation of the final classifica-

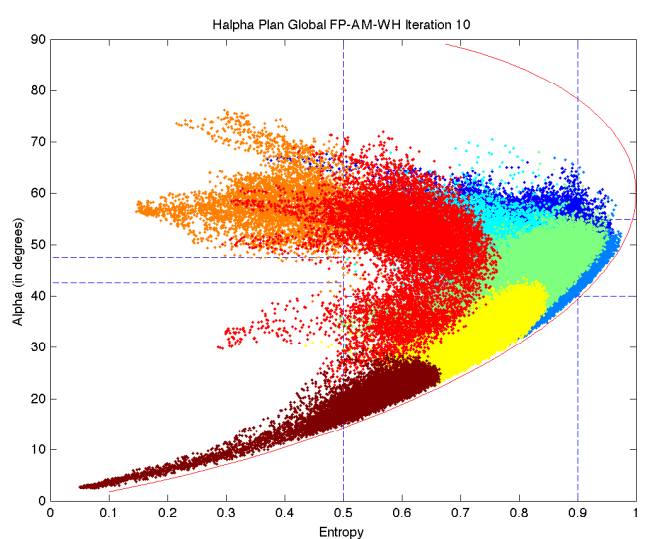

Fig. 3. Location of the pixel in the $\mathrm{H} / \alpha$ plan after 10 iterations in the SIRV case for the classical mean.

tion. The mean associated with the Riemannian metric corresponds to the geometric mean:

$$
\mathbf{M}_{\omega_{l}}=\underset{\mathbf{M}_{\omega} \in \mathcal{P}(m)}{\arg \min } \sum_{k=1}^{k}\left\|\log \left(\mathbf{M}_{k}^{l-1} \mathbf{M}_{\omega}\right)\right\|_{F}^{2},
$$

where $\|.\|_{F}$ stands for the Frobenius norm and $\mathcal{P}(m)$ is the set of the Hermitian definite-positive CM of size $m$. The solution $\mathbf{M}_{\omega_{l}}$ can easily be found using a simple gradient algorithm given by the following iterative procedure:

$\mathbf{M}_{k}=\mathbf{M}_{k-1}^{H / 2} \exp \left(\epsilon \sum_{i=1}^{K} \log \left(\mathbf{M}_{k-1}^{-H / 2} \mathbf{M}_{i}^{l} \mathbf{M}_{k-1}^{-1 / 2}\right)\right) \mathbf{M}_{k-1}^{1 / 2}$,

where $k \in \mathbb{N}^{*}$, where $\epsilon$ controls the speed of the gradient descent and where $\mathbf{M}_{0}=\mathbf{M}_{1}^{l}\left(\mathbf{M}_{1}^{l-1} \mathbf{M}_{2}^{l}\right)^{1 / 2}$ is, for example, the geometrical mean of the first two matrices.

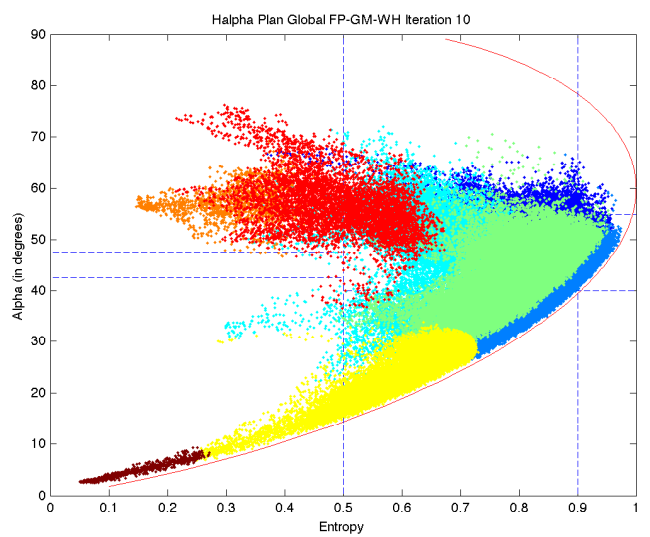

Fig. 4. Location of the pixels in the $\mathrm{H} / \alpha$ plan after 10 iterations in the SIRV case for the geometrical mean.

Figure 4 shows, after 10 iterations, the locations of all the pixels in the $\mathrm{H}-\alpha$ plan (for eight classes). Figure 5 repre- 
sents a comparison between the unsupervised $\mathrm{H} / \alpha$ classification results obtained with classical Arithmetical mean and the Riemannian mean (after 10 iterations) with the Fixed Point Covariance matrices.

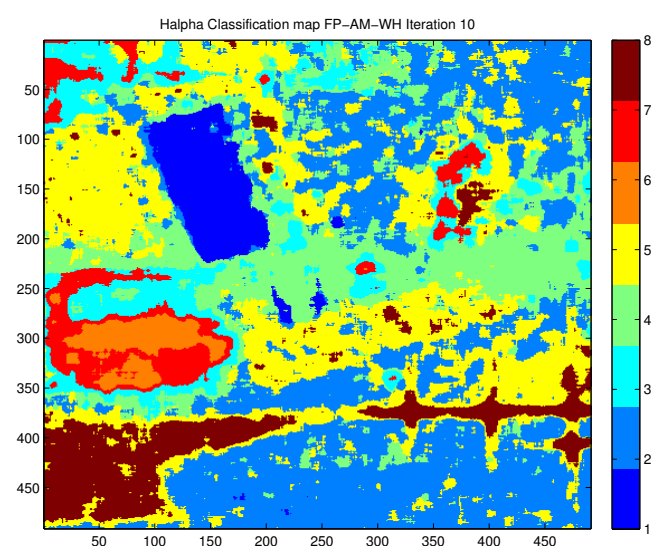

(a) Fixed Point and Arithmetical Mean

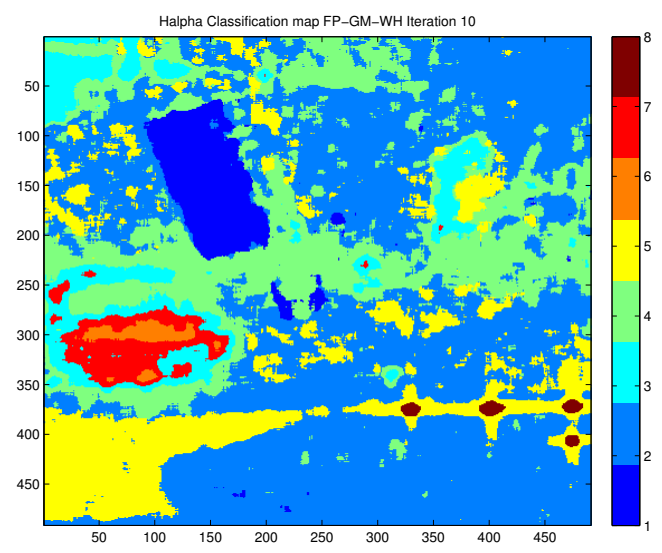

(b) Fixed Point and Geometrical Mean

Fig. 5. Comparison of unsupervised $\mathrm{H} / \alpha$ classifications after 10 iterations for arithmetical and geometrical mean

\section{CONCLUSION}

After recalling some recent techniques useful for modeling the heterogeneity and/or the non-Gaussian behavior of the polarimetric SAR images, this paper ${ }^{1}$ has presented the use of a new technique, based on the differential geometry, for computing the barycenter of $K$ Hermitian CM. This mean is used in the $\mathrm{H} / \alpha$ unsupervised process and allows, jointly with the Fixed Point Covariance Matrix estimate, to propose a new classification scheme. The latter seems promising (much more stable location in the $\mathrm{H} / \alpha$ plan) but needs however to be validated with ground truth.

\footnotetext{
${ }^{1}$ The authors would like to thank the DGA for supporting this research.
}

\section{REFERENCES}

[1] S. R. Cloude and E. Pottier, "An Entropy Based Classification Scheme for Land Applications of Polarimetric SAR", IEEE Trans. on Geoscience and Remote Sensing, Vol. 35, No. 1, pp.68-78, Jan. 1997.

[2] G. Vasile, J.P. Ovarlez, F. Pascal and C. Tison, "Coherency Matrix Estimation of Heterogeneous Clutter in High Resolution Polarimetric SAR Images", IEEE Trans. on Geoscience and Remote Sensing, Vol.48, No.4, pp.1809-1826, April 2010.

[3] V. Devlaminck and P. Terrier, "Geodesic Distance on nonSingular Coherency Matrix Space in Polarization Optics", J. Opt. Soc. Am. A, Vol.27, No.8, August 2010.

[4] M. Moakher, "Differential Geometric Approach to the Geometric Mean of Symmetric Positive-Definite Matrices", SIAM J. Matrix Anal. Appl., Vol.26, No.3, pp.735747, 2005.

[5] F. Barbaresco, "Innovative Tools for Radar Signal Processing Based on Cartan's Geometry of SPD Matrices and Information Geometry", IEEE International Radar Conference, Rome, May 2008.

[6] J. S. Lee, M. R. Grunes, T. L. Ainsworth, D. Li-Jen, D. L. Schuler, and S. R. Cloude, "Unsupervised Classification Using Polarimetric Decomposition and the Complex Wishart Classifier," IEEE Trans. on Geoscience and Remote Sensing, vol. 37, no. 5, pp. 2249-2258, 1999.

[7] J. S. Lee, M. R. Grunes, E. Pottier, and L. Ferro-Famil, "Unsupervised Terrain Classification Preserving Polarimetric Scattering Characteristics," IEEE Transactions on Geoscience and Remote Sensing, Vol. 42, No. 4, pp. 722731, 2004.

[8] F. Gini and M. V. Greco, Covariance Matrix Estimation for CFAR Detection in Correlated Heavy Tailed Clutter," Signal Processing, Vol. 82, No. 12, pp. 1847-1859, 2002.

[9] E. Conte, A. De Maio, and G. Ricci, "Recursive Estimation of the Covariance Matrix of a Compound-Gaussian Process and its Application to Adaptive CFAR Detection," IEEE Trans. on Image Processing, Vol. 50, No. 8, pp. 1908-1915, 2002.

[10] F. Pascal, Y. Chitour, J.P. Ovarlez, P. Forster and P. Larzabal, "Covariance Structure Maximum Likelihood Estimates in Compound Gaussian Noise: Existence and Algorithm Analysis", IEEE Trans. on SP, Vol. 56, No. 1, pp. 34-48, Jan. 2008.

[11] F. Pascal, P. Forster, J.P. Ovarlez and P. Larzabal, ”Performance Analysis of Covariance Matrix Estimates in an Impulsive Noise", IEEE Trans. on SP, Vol. 56, No. 6, pp. 2206-2217, Jun. 2008. 